\title{
Regional Brain Activations in Awake Unrestrained Dogs
}

\author{
Peter F. Cook ${ }^{\mathrm{a}}$, Andrew Brooks ${ }^{\mathrm{a}}$, Mark Spivak ${ }^{\mathrm{b}}$, Gregory S. Berns ${ }^{\mathrm{a}}$
}

a. Department of Psychology, 36 Eagle Row, Emory University, Atlanta, GA 30322 USA

b. Comprehensive Pet Therapy, 6600 Roswell Rd NE Atlanta, GA 30328 USA

pfcook@emory.edu (corresponding author), abrook6@emory.edu, markcpt@aol.com, gberns@emory.edu 


\section{Abstract}

Due to its accessibility, tractability, evolutionary history, and social intelligence, the domestic dog is increasingly used as a model animal in behavioral studies. While this has led to new insights into canine cognition, understanding of dog brain function has not advanced apace. Practical and ethical concerns have limited the use of the invasive brain imaging techniques typically used with primate and rodent models. Now, with the advent of awake, unrestrained canine fMRI (Berns, Brooks \& Spivak, 2012), data can be obtained on brain function during passive reception of stimuli and even during active decision making. Such data have already proven useful for linking brain regions with particular cognitive functions, illuminating canine socioemotional processes, and grounding behavioral observations of phenotypic variability in a neural substrate. Future studies may shed new light on brain networks and neural dysfunction in dogs, and will likely bear relevance for clinical practitioners, dog trainers, and comparative psychologists and neuroscientists. This brief review discusses prior dog imaging work; addresses the practical and technical hurdles to awake, unrestrained dog imaging; and discusses the current state of the science.

Key Words: brain imaging, comparative neuroscience, canine cognition, fMRI, dog behavior 


\section{$\underline{\text { Introduction }}$}

Dog behavioral science is undergoing a renaissance. Convergent interest in the dog as a companion, a working animal, and a comparative model for social psychology, has produced a burgeoning literature ranging from dogs' comprehension of human gestures to their olfactory sensitivity (e.g., Gacsi et al, 2009; Miklosi, 2014; Hare, Brown, Williamson, \& Tomasello, 2002; Udell, Dory \& Wynne, 2008; Williams \& Johnston, 2002). Given the widespread interest, both general and scientific, in dog behavior, it is striking how little we know about brain function specific to this species. Although fMRI approaches using sedation and restraint have provided some insight, particularly regarding basic sensory processes in dogs (Willis et al., 2001; Bach et al. 2013), chemical and physical restraint limit the types of neural processes that can be studied, and many researchers shy away from using these approaches in dogs due to ethical concerns.

As is manifestly clear from both human cognitive neuroscience and neurobehavioral primate and rodent models, functional MRI can validate and improve models of cognition and behavioral function. Brain imaging can situate animals in a comparative evolutionary framework and has a wealth of potential applications, including the quantification of neural dysfunction (For some of the applications and limitations of fMRI in basic science and clinical practice, see: Fox \& Greicius, 2010; Logothetis, 2008; Matthews, Honey \& Bullmore, 2006).

Because $\mathrm{fMRI}$ is very susceptible to subject motion, non-human animals are typically imaged under physical or chemical restraint. However, due to the ease of training dogs and advances in fMRI technology and analysis, Berns, Brooks \& Spivak (2012) showed that quality fMRI data could be obtained from dogs using a cooperative approach without restraint or sedation. More recently, Cook, Spivak \& Berns (2015) have shown that active-task paradigms, similar to human fMRI studies, are also achievable. Moreover, awake, unrestrained imaging sidesteps the practical and ethical limitations of prior approaches to exploring dog brain function, and a number of research groups using this approach have already provided new insights into the canine brain. Related findings will help inform the growing field of canine behavioral science and may also have practical implication for trainers, breeders, and clinicians.

To date, dog-fMRI studies have focused on reward systems and preference, olfaction, and the neural underpinnings of social cognition, and each of these research areas will be addressed in turn. Potential hurdles in training MRI dogs and in data processing and analysis will also be addressed. 


\section{Functional MRI Background}

Functional magnetic resonance imaging ( $F M R I)$ is the most widely used functional imaging technique in human cognitive neuroscience. Although the limitations of fMRI are widely recognized (Huettel, Song \& McCarthy, 2004), it remains the most broadly applicable and accessible non-invasive imaging approach able to achieve relatively high spatial and temporal resolution, and has led to landmark discoveries in perception, memory, and cognitive control. FMRI measures the blood oxygen level dependent (BOLD) neural response (Ogawa, Lee, Kay \& Tank, 1990). This is an approximation of firing activity across a large number of neurons. In brief, any mental process will involve an increase (or decrease) of firing activity across connected networks of neurons. An increase of activity has a metabolic cost, which is followed by a local increase in oxygenated blood to compensate. Because oxygenated and deoxygenated hemoglobin have different magnetic properties, fMRI imaging sequences are optimized to pick up on these subtle local changes in blood oxygenation, providing a proxy for neural activity.

Through careful experimental paradigms, one can measure the changes in brain activity that are timematched to the presentation of stimuli and the production of a limited number of behavioral actions. This, in turn, can allow one to match activity in particular regions with particular mental processes, helping to establish the neural underpinnings of cognition, emotion, and behavior.

Changes in BOLD signal accompanying neural activation tend to be quite small. Because the whole brain is constantly active to one degree or another, BOLD signal is also quite noisy. To address these limitations, typical fMRI paradigms average BOLD activation across multiple repetitions of a stimulus or condition. In addition, because most experimental conditions trigger a complex and multi-faceted neural response, $\mathrm{fMRI}$ paradigms need to use careful control conditions to isolate the specific mental processes of interest.

To illustrate, imagine one wanted to use fMRI to locate the early cortical substrate of vision (typically referred to as V1). One could have a subject periodically close and open his or her eyes, then subtract the mean BOLD signal across the brain during eyes closed from that during eyes open, i.e. the contrast, [open - closed]. The result would indicate brain regions where the BOLD signal was higher (and thus underlying neural activity was higher) when receiving visual stimulus versus not. Such experiments have been done, and they reliably show a region in posterior occipital cortex where we know the visual signal first reaches cortex in humans. However, if one instead wanted to examine the neural substrate of motion vision, the control condition could no longer be as simple as the subjects' closing their eyes. 
Instead, you would need to show simple moving stimuli, and, as a control, static versions of the same images.

Importantly, most fMRI studies do not focus on results from an individual subject. Instead, they average many individual results together and look for common areas of condition-specific activation across subjects. For example, if one wanted robust evidence that $\mathrm{V} 1$ tends to share its location across different individuals, one could repeat the above experiment with a number of subjects. The next step is "normalization," or spatial transformation, in which the brain images from each subject are warped to fit a common template brain space. Then one could average the result of the [open - closed] contrast across all subjects, and subject it to statistical tests in the group template space. Achieving a statistically robust, condition-specific regional activation at the group level is considered the standard for most fMRI research with humans.

Also of note, although $\mathrm{fMRI}$ has better spatial or temporal resolution than other common techniques for assessing brain function - such as electroencephalogram (EEG), single-photon emission computerized tomography (SPECT) and positron emission tomography (PET) - the resolution is still a significant constraint. Typical human imaging studies feature voxels (three dimensional pixels, the base unit of resolution in 3D imaging) with dimensions ranging between 1 and $3 \mathrm{~mm}$. A one $\mathrm{mm}$ cube of cortex can contain more than 100,000 neurons, and most statistically robust activations in fMRI studies involve multiple contiguous voxels. That means that $f M R I$ is able to probe only regional contributions to mental processes that take up a relatively extensive and contiguous portion of the brain. In the time domain, a neuron can fire over 200 times a second, but the BOLD response aggregates over multiple neurons, and is fairly slow, lagging 4-6 seconds behind the actual neural activation, taking a few seconds to peak and then decline. Although recent advances in fMRI imaging acquisition can decrease the acquisition time under a second, in most applications the temporal resolution for resolving mental processes in $\mathrm{fMRI}$ is still on the order of seconds (Huettel, Song \& McCarthy, 2004).

\section{Prior Work on Dog Brain Function}

As with other neurobehavioral animal models, there is a history of invasive brain lesion work with dogs aimed at uncovering the function of different brain regions (e.g., Culler, \& Mettler, 1934; Fuller, Rosvold \& Pribram, 1957; Brutkowski \& Dabrowska, 1966). In such studies, a part of the brain is damaged, and then changes in behavior are measured, allowing inferences about the relationship of the damaged 
region to the measured behavior (Rorden \& Karnath, 2004). However, such studies used fairly crude lesion methods, never reached the ubiquity of parallel research in rodents and primates, and fell out of favor for use in canines in the latter part of the $20^{\text {th }}$ century for ethical reasons.

More recent work on local brain function in the dog has been predominately clinical in nature, and has come by way of brain imaging under restraint or sedation (to control for subject motion, which is a major confound for functional imaging). FMRI studies have examined pain response (Chang et al., 2012), emesis (Yu et al., 2014), and dysfunction in vision (Willis et al., 2001; Gingras et al.; 2009) and auditory pathways (Bach et al., 2013).

Also used in the clinical setting with dogs is single-photon emission computed tomography (SPECT), which involves injecting subjects with radio-tracers that preferably bind with particular tissue types in the body (LeBlanc \& Peremans, 2014). This in turn allows assessment of brain regions that show increased or decreased accumulation of these tracers over time. Depending on the tracers used, general brain activity or activity of specific neurotransmitter systems can be assessed. SPECT cannot achieve the spatial or temporal resolution of fMRI, and is typically used to obtain a functional "snapshot" of brain activity during a particular condition. SPECT studies in dogs have examined brain regions and neurotransmitter systems involved in canine anxiety and aggression (Vermeire et al, 2009; Vermeire et al, 2011; Vermeire et al, 2012) and epilepsy (Martle et al, 2009).

While imaging under physical and chemical restraint has been used in dogs predominately for clinical applications, there is precedent in other model species such as rodents and primates for using these same approaches to explore a wider range of neurobehavioral processes (Orban, 2002; Logothetis, 2003; Tsao, Freiwald, Tootell \& Livingstone, 2006, Keilholz et al., 2004; Brockmann, Kemmling \& Groden, 2007; Vanduffel, Zhu \& Orban, 2014).

Much has been learned with imaging in restrained rodents and primates, particularly in regard to visual processing in the brain. However, chemical and physical restraints come with a cost-not only are there ethical concerns, but the direct effects of various anesthetics and the indirect effects of stress from the physical restraints can compromise imaging data in a variety of ways and limit the types of experiments one can conduct.

Extensive study into commonly used veterinary anesthetics has shown that many of them blunt or alter the BOLD response across multiple brain regions (Antognini, Buonocore, Disbrow \& Carstens, 1997; Peeters, Tindemans, De Schutter \& Van der Linden, 2001; Peltier et al., 2005; Plourde et al., 2006; Qiu et 
al., 2008; Liu et al., 2013), presenting an obvious confound for interpretation of functional images obtained under anesthesia. Some agents, given at sub-anesthetic doses, may allow image acquisition without broadly disrupting the BOLD response. Abel et al. (2003) found limited evidence of BOLD disruption with low-dose keteamine, although Waelbers et al. (2015) report that use of ketamine as an additional anesthetic affected regional perfusion as measured with SPECT. Of note, some neural processes, most notably early vision (Goense \& Logothetis, 2008), are somewhat maintained under anesthesia. Some hypotheses about brain function can be meaningfully tested with sedation or anesthetic models (Van der Linden, Van Camp, Ramos-Cabrer \& Hoehn, 2007).

Use of physical restraint without anesthesia is another option, and various restraint protocols have been developed for use in rodent and primate scanning (e.g., Sachday et al., 2003; Ferris \& Snowdon, 2005). However, use of restraint has ethical implications, and induces anxiety that can affect BOLD signal and must be managed (King et al., 2005).

None of these confounds are, by themselves, insurmountable. But they can be neatly sidestepped through the use of domestic dogs trained to remain still in the scanner during data acquisition. In addition, imaging without anesthesia or restraint allows dogs to use active sensory processes (e.g., sniffing) or even produce motor responses in active-task paradigms.

\section{Challenges in Imaging Awake Dogs}

While the potential benefits, both ethical and theoretical, to imaging awake unrestrained dogs are clear, there are procedural and technical challenges. To date, three research groups have published results obtained in awake unrestrained dogs, and the methods of each group have varied. Reassuringly for others interested in pursuing dog imaging, each group has been successful in producing plausible and informative data.

A great benefit of neurobehavioral work with dogs is their availability. Indeed, two research groups have used a citizen science approach, successfully recruiting participants from the local domestic dog population (Berns, Brooks \& Spivak, 2012; Andics et al, 2014).

After acquiring access to subjects, implementing a comprehensive training program is the next step. FMRI data is highly susceptible to pollution from subject motion. Without restraint or sedation, dogs are free to move within the scanner, so they must be trained to hold very still for the amount of time 
necessary for image acquisition (varying from 30 seconds to 5 minutes depending on study design). In addition, live scanning presents a potentially stressful environment, with repetitive noises up to $100 \mathrm{~dB}$ and consistent vibrations, all within the scanner bore, which is an elevated and constricted tube.

Training for motionlessness has been broadly similar across research groups, using successive approximation of duration in a down-stay with positive reinforcement, and also, in one case, social learning (Andics et al., 2014). One group has also used custom-designed and fitted foam chin rests that promote stable positioning (Berns, Brooks \& Spivak, 2014).

Desensitization to the scanner environment has also been similar across groups, with praise and reward delivered following increasing duration in the actual scanner (Andics et al., 2014; Jia et al., 2014) or in a custom-designed simulator using recorded scanner noise (Berns, Brooks \& Spivak, 2012). One group has also used hearing protection for each subject (Berns, Brooks \& Spivak, 2012), which has also required typical desensitization procedures.

The end point of this training has been that, across all three research groups, a large number of dogs have successfully participated in live scanning. Given this, and the domestic dog's trainability and general cooperativeness, it is clear that careful application of typical animal training approaches enables successful fMRI data acquisition with domestic dogs. Importantly, these approaches have not been used to date with behaviorally disordered dogs such as may be encountered in clinical practice. More work is required to assess the feasibility awake $\mathrm{fMRI}$ with that canine population.

On the technical side, the two primary challenges to scanning awake unrestrained dogs are motion (even the best trained dogs move more than typical humans during live scanning) and image normalization (that is, transforming the spatial characteristics of acquired images from individual dogs so that they can be combined and analyzed at the group level). Here, the approaches of the different research groups have diverged more greatly than in behavioral training.

Motion is a particular concern for fMRI. If a subject moves, and is thus in a different position in one image than in a prior, changes in BOLD signal from different parts of the brain will be superimposed. In addition, moving can create artifacts that can increase or decrease measured BOLD signal. The typical motion correction approach used in human imaging is to take each sequence of functional images and use software algorithms to realign (i.e., reposition) each image in the sequence to one target image from within the sequence. Each research group has used the built-in motion correction tools in the imaging software they've used to do just this. In addition, because initial statistical results in fMRI are 
computed with a general linear model (GLM) across all voxels in the brain, it is common to include a time course of motion (in each of the three cardinal directions and rotation) as a nuisance regressor, such that artifactual changes in BOLD signal that can be simply explained as due to motion are regressed out of the results of interest. All three groups also followed this standard procedure, computing motion time courses from the realignment parameters and including them in their respective GLMs.

Two of the research groups also used additional approaches to motion correction that are less standard in human imaging. Jia et al. (2014) developed a scanner-independent motion tracking camera system that allowed them to generate motion time courses independent from the image realignment parameters, and then enter these as additional nuisance variables in the GLM analysis. This video-based approach is technically non-trivial, but may produce more precise movement parameters. Berns, Brooks \& Spivak (2012) developed an approach relying on aggressive "censoring" of the fMRI images. Censoring involves proactively removing volumes/time points from the fMRI image series that are corrupted by motion prior to GLM analysis (Stower et al., 2012). This can be particularly useful for imaging protocols that include reliable motion, such as when the dog is consuming a food reward, or making a motor response. Berns, Brooks \& Spivak (2012) used preset criteria to determine which images would be censored. Following image realignment, individual images (volumes) were removed from the scan sequence either if the image-to-image motion exceeded $1 \mathrm{~mm}$ in any of the three cardinal directions or if the BOLD signal averaged across the entire brain changed by more than $1 \%$ in one image from the directly preceding image (indicative of a large head movement). The resulting data sets were cleaner, although they had to be manually assessed to be certain that enough images remained within the conditions of interest to allow for valid statistical analysis.

While motion correction involves ensuring that each image within an $\mathrm{fMRI}$ sequence is in the same spatial coordinates for individual subjects, a related concern is group registration, in which the statistical results from individual subjects are all transformed into the same group template space (e.g., Smith et al, 2004). In humans, and other primates, there are standard and widely used template brain atlases, but this is not yet the case for dogs. Therefore, each research group has used a different template as a target for group registration: a high resolution structural scan from an individual dog (Jia et al., 2014), an average of structural images from dogs in the study (Andics et al., 2014), and a high resolution template brain created by averaging together a number of structural scans (Berns, Brooks \& Spivak 2013; Datta et al., 2012). When averaging together multiple structural scans to produce a template, some local 
anatomical information useful that may aid registration is lost. In tradeoff, the resulting template may be more forgiving of the individual and breed differences found in dog brain morphology.

Each group employed a similar standard registration workflow, registering individual functional images to an intermediary, and then from there to the template space, but there were notable differences in exact procedure and tools used. While two groups registered individual functional images to individual structural images, and then registered the result to template space (Andics et al., 2014; Berns, Brooks \& Spivak, 2013), the third group registered individual functional scans to the individual functional scan of one anesthetized dog, and from there to the individual structural scan of that dog (Jia et al., 2014). In addition, while two of the groups used the standard registration algorithms included in the SPM imaging suite (Andics et al., 2014; Jia et al., 2014), the third used the Advanced Neuroimaging Tools (ANTs) toolbox (Berns, Brooks \& Spivak, 2013; Avants et al., 2011).

More generally, while the scan parameters used in image acquisition have varied from experiment to experiment and from group to group, resolution for functional scans has remained consistently in the 3 $\times 3 \times 3 \mathrm{~mm}$ range, and acquisition time in the 1-2 second range, which represent a reasonable tradeoff between spatial and temporal resolution given the likelihood of motion with awake, unrestrained dogs.

\section{Experiments to Date}

FMRI of awake unrestrained dogs is still an emerging field, but the technique has already enhanced our understanding of dog neuro- and sociobiology. The published studies (Table 1) show a surprising diversity of experimental methods. Some have used passive exposure to sensory stimuli while others have examined neural response to stimuli with previously trained associations. One study in review (Cook, Spivak \& Berns) has even used an active-task paradigm, in which dogs were required to produce or withhold a motor response. Studies are also divided between those using a blocked presentation design (where multiple stimuli of one type are presented consecutively before switching to a block of another stimulus type) and an event-related design (in which stimuli representing different conditions are presented in semi-random order) (Amaro \& Barker, 2006). 
Table 1: Published Studies Using Awake Dog fMRI

\begin{tabular}{|c|c|c|}
\hline Study & Topic & Modality \\
\hline $\begin{array}{l}\text { Berns, Brooks \& Spivak, } \\
2012\end{array}$ & Conditional reward & Event-based passive presentation \\
\hline $\begin{array}{l}\text { Berns, Brooks \& Spivak, } \\
2013\end{array}$ & Conditional reward & Event-based passive presentation \\
\hline Andics et al., 2014 & $\begin{array}{l}\text { Auditory processing - human and } \\
\text { dog vocalizations }\end{array}$ & Block-based passive presentation \\
\hline $\begin{array}{l}\text { Cook, Spivak \& Berns, } \\
2014\end{array}$ & $\begin{array}{l}\text { Conditional reward - social and non- } \\
\text { social sources }\end{array}$ & Event-based passive presentation \\
\hline Jia et al., 2014 & Olfactory processing & Block-based passive presentation \\
\hline $\begin{array}{l}\text { Berns, Brooks \& Spivak, } \\
2015\end{array}$ & $\begin{array}{l}\text { Olfactory processing - human and } \\
\text { dog scents }\end{array}$ & Event-based passive presentation \\
\hline Dilks et al., 2015 & Face processing & Block-based passive presentation \\
\hline $\begin{array}{l}\text { Cook, Spivak \& Berns, } \\
\text { Under Review }\end{array}$ & Self control & Event-based active task paradigm \\
\hline
\end{tabular}

Stimuli used have been visual, olfactory, and auditory, and visual stimuli have been presented both on screen and by a person in view of the dog. In other words, dog scanning allows for much of the flexibility in experimental paradigm that is found in human imaging studies.

In the first paper published with awake, unrestrained dogs, Berns, Brooks \& Spivak (2012) trained two dogs out of the scanner to associate one hand signal with incipient food reward, while a second hand signal predicted no reward receipt. In the scanner, both dogs showed a significant, differential increase of brain activation in the ventral striatum when viewing the reward signal versus the no-reward signal. The role of the ventral striatum in reward prediction is well established across multiple species (Schultz, Apicella, Scarnati \& Ljungberg, 1992; Pagnoni, Zink, Montague \& Berns, 2002; van der Meer \& Redish, 2011), so this result represented a meaningful validation of the general procedure, including motion correction and individual subject normalization procedures. In a follow-up, Berns, Brooks \& Spivak (2013) replicated the finding across 11 dogs, obtaining a significant group-level result. This further 
validated the basic approach, and showed the feasibility of accurate group registration (non-trivial given the high variability in dog brain morphology).

Subsequent studies from this research group have focused on reward center activation, using it as a tool to delve into dog sociobiology and individual variability. In Cook, Spivak \& Berns (2014), the same reward and no-reward signals were used across 12 dogs, but signals were presented in three separate conditions: from the familiar handler, from an unfamiliar person, and as cartoon images on a computer screen. Again, dogs showed a robust ventral striatal activation at the group level. Interestingly, a strong difference in relative response to reward versus no reward signals was seen between dogs ranked either low or high for aggressive behavior on the CBARQ scale of canine temperament (van den Berg, Heuven, van den Berg, Duffy \& Serpell, 2010). Low aggressivity dogs showed a much higher differential response to the two hand signals when presented by the familiar handler, while high aggressivity dogs showed a much higher differential response to the two hand signals when presented by the unfamiliar person or computer. This suggests that the source of information affects its relative reward value and salience for the dog, and the manner in which it does so depends on temperament. In a complementary finding, Berns, Brooks \& Spivak (2015) presented odor samples to 12 dogs and found that ventral striatal response was much higher in dogs when smelling the odor of a familiar human than that of an unfamiliar human, or that of a familiar or unfamiliar dog. Of note, the familiar humans who served as scent sources for this study were not the dog's primary handlers and were not present for scanning, suggesting that dogs: a). recognized the scent of a familiar human, and b) had a positive association with it that they do not show for familiar dogs. One of the great benefits of using reward prediction paradigms is that caudate activation can be taken as an analog to positive association and preference, allowing direct assessment of the emotional and motivational world of dogs beyond what can be discovered via behavioral approaches alone.

In another paper using olfactory stimuli, Jia et al (2014) presented both anesthetized and awake dogs with low and high concentrations of odorants. They showed that, although primary olfactory cortex was activated in both awake and anesthetized dogs, cortical areas showing differential activation to odors were only discernible in awake dogs, emphasizing the value of awake imaging. Studies such as these may pave the way to more nuanced examinations of the biological substrate of odor detection and processing in canines, which may help inform training and use of scent detection dogs.

Much has been made in the behavioral literature about dogs' extreme sensitivity to human social cues and communication (pointing, facial expression). The advent of awake canine imaging now allows us to 
further assess the neurobiological substrate for dog social cognition. Andics et al. (2014), using a novel comparison between human $(n=22)$ and canine $(n=11)$ imaging results, showed apparent similarities in the regions activated differentially by vocal stimuli versus non-vocal auditory stimuli. Human and dog subjects were both exposed to human and dog vocalizations, non-vocal sounds, and a silent baseline. In addition, the vocalizations of both humans and dogs ranged in valence from positive to negative. Dogs, unlike humans, showed fairly extensive auditory regions with greater activation for dog versus human vocalizations. In addition, not only did dogs, like humans, show secondary auditory regions that were maximally sensitive to the human voice, both dog and human subjects also showed greater activation in certain auditory regions for positively versus negatively valenced vocal stimuli. The authors suggest that these results, the first showing conspecific-vocalization regions in a non-primate, raise the possibility that vocalization-specific auditory brain regions may have been present in the common ancestor of carnivores and primates, 100 million years ago.

In a study examining neural response to face stimuli, Dilks et al. (2015) showed differential activation to faces versus objects in a region of ventral caudal temporal lobe that may be roughly analogous to fusiform gyrus in primates. Dogs were first scanned while watching movie stimuli of faces, objects, and other visual categories. For each dog, a region in temporal lobe showing greatest differential activation to faces versus objects was identified. Then, in a second set of presentations, dogs were shown static images of dog and human faces, objects, and other visual categories. Relative activation in the previously identified region to the static face images (both dog and human) was significantly greater than to the static object images. Results from this test/re-test approach are strongly indicative of a facespecific processing region in the ventral visual stream in dogs, analogous to the widely studied primate fusiform face area (FFA). Whether this is the product of dogs' evolutionary relationship to humans, or is emblematic of a wide distribution of face-specific processing adaptations in mammals remains to be seen, but it does emphasize the importance of face perception for dogs, and paves the way for future studies examining neurological response to facial expression and related phenomena. In conjunction with the Berns Brooks \& Spivak (2015) olfaction study and the Andics et al. (2014) study showing auditory regions activated specifically by human vocalizations, these findings suggest a fairly high degree of specialization in dog sensory cortex for human social signals, across multiple modalities, and are in line with behavioral findings highlighting dogs' unique sociobiological faculties.

This early work fMRI work has delved into subcortical reward regions, and sensory processing-even less is known about frontal regions and association cortex in the dog. In Cook, Spivak \& Berns (under 
review), dogs were trained on a go/no-go task out of the scanner, and then scanned. Although BOLD signal could not be measured during movement, comparison of brain activity on successful inhibition trials versus neutral trial types showed increased activity in a region of premotor and frontal cortex, mirroring prior findings in primates. In addition, brain activation in this region correlated with fewer false alarms in the scanner, which correlated with better performance on a self-control test out of the scanner. In addition to mapping out a previously unexplored region of dog cortex, and showing its dynamic relationship with self-control behavior, this study demonstrated the feasibility of doing activetask scanning paradigms with dogs in an fMRI environment.

\section{Conclusion and Future Directions}

The dog has proven an able contributor to unrestrained awake brain imaging studies. Multiple groups using multiple methodologies have demonstrated the validity of this approach, and, despite the early stages of the field, findings are already deepening our knowledge of dog behavior, sociobiology, and sensory systems. With appropriate training protocols, and careful application of motion correction and normalization procedures, there should be no great impediment for research groups newly interested in obtaining dog imaging data.

Dog fMRI may show great benefit in the clinical setting, particularly in helping to explore phenotypic neural differences associated with disease states. Although it does not allow direct imaging of transmitter systems as do nuclear approaches (e.g., PET and SPECT), it allows high resolution regional and network-level analysis of typical and altered brain function that can be cross-referenced to findings using other imaging modalities. Two primary hurdles remain. First, despite the high success rate of training dogs for $\mathrm{fMRI}$ in the research setting, current methodologies take weeks or months, and may be most successful with dogs showing high drive and a generally calm temperament. New training approaches may be required to enact clinical imaging of pet dogs with limited prior training experience and less suitable dispositions. For high anxiety dogs, use of anxiolytics may also be a solution when clinically warranted. Second, dog brain morphology is heterogeneous. To date, the majority of dogs taking part in awake $\mathrm{fMRI}$ have been retrievers, labs, or border collies. More work may be required to develop optimal normalization approaches for use in cohorts with greater diversity in brain morphology. In a parallel concern, cross-study comparison of regional brain activation benefits greatly from use of a standardized common brain atlas. Given the differences in brain morphology across breeds, it is an open question how best to develop such an atlas for use across diverse dog breeds. 
The field of canine neuroimaging is currently wide open, but a number of research questions may be of particular theoretical interest. Studies discussed here suggest that the dog has neurobiological adaptations supporting social perception and intelligence, but there's much more work to be done assessing neurobehavioral components to social intelligence in the domestic dog. New behavioral findings suggest dogs may feel jealousy (Harris \& Prouvost, 2014) and they respond differentially to individuals based on how they interact with the dogs' owners (Chijiiwa et al., 2015). Clearly dogs have a complex social ecology, involving both other dogs and humans. Awake fMRI paradigms allow us to probe the neural underpinnings of social intelligence and compare them to what's known in other social species such as primates. This may help us answer questions regarding the uniqueness of dogs' social faculties among canid carnivores.

Studies of sensory neurobiology are also well suited to fMRI approaches. In the dog, research with fMRI has already probed the visual, auditory, and olfactory domains. Work to date has shown the social importance of all these senses for the dog and has begun to map out the neurological substrates. Future studies might decode neural representations of sensory stimuli and examine the neural processes driving integration of representations across sensory modalities. This work would help us better understand the sensory world of the dog, and could contribute to ongoing attempts to enhance selection, training and application of working dogs across multiple applications. Also on the applied front, studies of preference and temperament may be useful for pinpointing optimal phenotypes for different types of training and applications (such as assistance dogs), and may help to inform ongoing behavioral work assessing different approaches to dog training (e.g., Rooney \& Cowan, 2011; Fugazza \& Miklosi, 2015).

More generally, the demonstrated ability to use a wide range of experimental paradigms opens the possibility for extensive and careful functional mapping of the cortex. The dog brain is grossly quite different from the primate and rodent brain-which regions are involved in decision making, affect, and self-control, and what sorts of neural pathology can impact these regions? In addition, as opposed to the primate and rodent literature, there is essentially no work on functional and structural connectivity in the dog brain. Brains are networks, and understanding the geography and dynamics of those networks is essential to understanding how brains function and dysfunction. A whole other set of hurdles and challenges would be involved in applying network analysis techniques in awake, unrestrained dogs such as resting state fMRI (Van Den Heuvel \& Pol, 2010) and diffusion tensor imaging (Le Bihan et al., 2001), but related advances in understanding dog neurobiology could be significant. 
Imaging awake, unrestrained dogs is a practical and ethical approach to comparative neuroscience. It is also timely. Dog behavioral science has expanded greatly, but research into the dog brain is still in its infancy. Early results show great promise for dog fMRI, and future research promises to inform clinical practice and further illuminate canine neurobiology.

\section{$\underline{\text { Acknowledgments }}$}

This work was supported by a grant from the Office of Naval Research (N00014-13-1-0253). Figures are adapted from prior publications. Figs. 1, 2, 3, and 5 are reused under the creative commons license (http://creativecommons.org/licenses/by/4.0/legalcode). Fig. 4 is reused under license from Elsevier, \#3724300854311.

\section{Conflict of interest statement}

Gregory Berns and Mark Spivak own equity in Dog Star Technologies and developed technology used in some of the research described in this paper. The terms of this arrangement have been reviewed and approved by Emory University in accordance with its conflict of interest policies. Andrew Brooks and Peter Cook do not have any conflict of interests.

\section{Authorship statement}

The idea for the paper was conceived by PC, AB, MS, and GB. Literature review was conducted by PC. Discussion of training methodologies was based on discussions between PC, MS, and GB. Discussion of technological methodologies was based on discussions between PC, AB, and GB. PC wrote the paper with contributions from MS and GB. All authors have approved the final article.

\section{References}

Abel, K. M., Allin, M. P., Kucharska-Pietura, K., Andrew, C., Williams, S., David, A. S., \& Phillips, M. L. 2003. Ketamine and fMRI BOLD signal: distinguishing between effects mediated by change in blood flow versus change in cognitive state. Hum. Brain. Mapp.18, 135-145.

Amaro, E., \& Barker, G. J. 2006. Study design in fMRI: basic principles. Brain Cogn. 60, 220-232.

Andics, A., Gácsi, M., Faragó, T., Kis, A., \& Miklósi, Á. 2014. Voice-sensitive regions in the dog and human brain are revealed by comparative fMRI. Curr. Biol. 24, 574-578.

Antognini, J. F., Buonocore, M. H., Disbrow, E. A., \& Carstens, E. 1997. Isoflurane anesthesia blunts cerebral responses to noxious and innocuous stimuli: a fMRI study. Life Sci. 61, PL349-PL354. 
Avants, B. B., Tustison, N. J., Song, G., Cook, P. A., Klein, A., \& Gee, J. C. 2011. A reproducible evaluation of ANTs similarity metric performance in brain image registration. Neuroimage 54, 2033-2044.

Bach, J. P., Lüpke, M., Dziallas, P., Wefstaedt, P., Uppenkamp, S., Seifert, H., \& Nolte, I. 2013. Functional magnetic resonance imaging of the ascending stages of the auditory system in dogs. BMC Vet. Res. 9, 210.

Berns, G. S., Brooks, A. M., \& Spivak, M. 2012. Functional MRI in awake unrestrained dogs. PLoS One 7, e38027.

Berns, G. S., Brooks, A., \& Spivak, M. 2013. Replicability and heterogeneity of awake unrestrained canine $\mathrm{fMRI}$ responses. PLoS One 9, e81698.

Berns, G. S., Brooks, A. M., \& Spivak, M. 2015. Scent of the familiar: An fMRI study of canine brain responses to familiar and unfamiliar human and dog odors. Behav. Processes 110, 37-46.

Brockmann, M. A., Kemmling, A., \& Groden, C. 2007. Current issues and perspectives in small rodent magnetic resonance imaging using clinical MRI scanners. Methods 43, 79-87.

Brutkowski, S., \& Dabrowska, J. 1966. Prefrontal cortex control of differentiation behavior in dogs. Acta Biol. Exp. 26, 425-439.

Chang, S. K., Jahng, G. H., Lee, S. H., Choi, I. W., Choi, C. B., \& Choi, W. S. 2012. Differential localization of pain-related neural responses during acupuncture stimulation using blood oxygen level dependent (BOLD) fMRI in a canine model. Am. J. Chin. Med. 40, 919-936.

Chijiiwa, H., Kuroshima, H., Hori, Y., Anderson, J. R., \& Fujita, K. (2015). Dogs avoid people who behave negatively to their owner: third-party affective evaluation. Anim. Behav. 106, 123-127.

Cook, P. F., Spivak, M., \& Berns, G. S. 2014. One pair of hands is not like another: caudate BOLD response in dogs depends on signal source and canine temperament. PeerJ 2, e596.

Cook, P. F., Spivak, M., \& Berns, G. S. (under review). Task-based fMRI in Awake Dogs Reveals Frontal Regulation of Self-control.

Culler, E. L. M. E. R., \& Mettler, F. A. 1934. Conditioned behavior in a decorticate dog. J. Comp. Psychol. 18, 291.

Datta, R., Lee, J., Duda, J., Avants, B. B., Vite, C. H., Tseng, B., ... \& Aguirre, G. K. 2012. A digital atlas of the dog brain. PloS One 7, e52140. 
Dilks, D. D., Cook, P., Weiller, S. K., Berns, H. P., Spivak, M. H., \& Berns, G. 2015. Awake fMRI reveals a specialized region in dog temporal cortex for face processing. PeerJ 3, e1115.

Diksic, M., Nagahiro, S., Chaly, T., Sourkes, T. L., Yamamoto, Y. L., \& Feindel, W. 1991. Serotonin synthesis rate measured in living dog brain by positron emission tomography. J. Neurochem. 56, 153162.

Ferris, C. F., \& Snowdon, C. T. 2005. Functional magnetic resonance imaging in conscious marmoset monkeys: methods and applications in neuroscience research. The Laboratory Primate: Handbook of Experimental Animals. Amsterdam: Elsevier Science.

Fox, M. D., \& Greicius, M. 2010. Clinical applications of resting state functional connectivity. Front. Syst. Neurosci. 4.

Fugazza, C., \& Miklósi, Á. (2015). Social learning in dog training: the effectiveness of the Do as I do method compared to shaping/clicker training. Appl. Anim. Behav. Sci. 171, 146-151.

Fuller, J. L., Rosvold, H. E., \& Pribram, K. H. 1957. The effect on affective and cognitive behavior in the dog of lesions of the pyriform-amygdala-hippocampal complex. J. Comp. Physiol. Psychol. 50, 89.

Gácsi, M., Gyori, B., Virányi, Z., Kubinyi, E., Range, F., Belényi, B., \& Miklósi, Á. 2009. Explaining dog wolf differences in utilizing human pointing gestures: selection for synergistic shifts in the development of some social skills. PLoS One 4, e6584.

Gingras, G., Komaromy, A. M., Tseng, B., Alexander, J. J., Chiodo, V. V., Hauswirth, W. W., ... \& Aguirre, G. K. 2009. Cortical recovery following gene therapy in a canine model of achromatopsia. J. Vis. 9, 311.

Goense, J. B., \& Logothetis, N. K. 2008. Neurophysiology of the BOLD fMRI signal in awake monkeys. Curr. Bio. 18, 631-640.

Hare, B., Brown, M., Williamson, C., \& Tomasello, M. 2002. The domestication of social cognition in dogs. Science 298, 1634-1636.

Harris, C. R., \& Prouvost, C. Jealousy in Dogs. PLoS ONE 9, e94597.

Huettel, S. A., Song, A. W., \& McCarthy, G. 2004. Functional Magnetic Resonance Imaging, Sunderland, MA.

Jia, H., Pustovyy, O. M., Waggoner, P., Beyers, R. J., Schumacher, J., Wildey, C., ... \& Deshpande, G. 2014. Functional MRI of the olfactory system in conscious dogs. PloS one 9.

Keilholz, S. D., Silva, A. C., Raman, M., Merkle, H., \& Koretsky, A. P. 2004. Functional MRI of the rodent somatosensory pathway using multislice echo planar imaging. Magn. Reson. Med. 52, 89-99. 
King, J. A., Garelick, T. S., Brevard, M. E., Chen, W., Messenger, T. L., Duong, T. Q., \& Ferris, C. F. 2005. Procedure for minimizing stress for fMRI studies in conscious rats. J. Neurosci. Meth. 148, 154160.

LeBlanc, A. K., \& Peremans, K. 2014. PET and SPECT imaging in veterinary medicine. Semin. Nucl. Med. 44, 47-56.

Le Bihan, D., Mangin, J. F., Poupon, C., Clark, C. A., Pappata, S., Molko, N., \& Chabriat, H. 2001. Diffusion tensor imaging: concepts and applications. J. Magn. Reson. Imaging 13, 534-546.

Logothetis, N. K. 2008. What we can do and what we cannot do with fMRI. Nature, 453, 869-878.

Liu, J. V., Hirano, Y., Nascimento, G. C., Stefanovic, B., Leopold, D. A., \& Silva, A. C. 2013. fMRI in the awake marmoset: somatosensory-evoked responses, functional connectivity, and comparison with propofol anesthesia. Neuroimage 78, 186-195.

Martlé, V., Peremans, K., Audenaert, K., Vermeire, S., Bhatti, S., Gielen, I., ... \& Van Ham, L. 2009. REGIONAL BRAIN PERFUSION IN EPILEPTIC DOGS EVALUATED BY TECHNETIUM-99m-ETHYL CYSTEINATE DIMER SPECT. Vet. Radiol. Ultrasound 50, 655-659.

Matthews, P. M., Honey, G. D., \& Bullmore, E. T. 2006. Applications of fMRI in translational medicine and clinical practice. Nat. Rev. Neurosci. 7, 732-744.

Miklósi, Á. 2014. Dog behaviour, evolution, and cognition. Oxford University Press.

Ogawa, S., Lee, T. M., Kay, A. R., \& Tank, D. W. 1990. Brain magnetic resonance imaging with contrast dependent on blood oxygenation. Proc. Natl. Acad. Sci. U. S. A. 87, 9868-9872.

Orban, G. A. 2002. Functional MRI in the awake monkey: The missing link. J. Cogn. Neurosci. 14, 965969.

Pagnoni, G., Zink, C. F., Montague, P. R., \& Berns, G. S. 2002. Activity in human ventral striatum locked to errors of reward prediction. Nat. Neurosci. 5, 97-98.

Peeters, R. R., Tindemans, I., De Schutter, E., \& Van der Linden, A. 2001. Comparing BOLD fMRI signal changes in the awake and anesthetized rat during electrical forepaw stimulation. Magn. Reson. Imaging 19, 821-826.

Peltier, S. J., Kerssens, C., Hamann, S. B., Sebel, P. S., Byas-Smith, M., \& Hu, X. 2005. Functional connectivity changes with concentration of sevoflurane anesthesia. Neuroreport 16, 285-288. 
Plourde, G., Belin, P., Chartrand, D., Fiset, P., Backman, S. B., Xie, G., \& Zatorre, R. J. 2006. Cortical processing of complex auditory stimuli during alterations of consciousness with the general anesthetic propofol. Anesthesiology 104, 448.

Qiu, M., Ramani, R., Swetye, M., Rajeevan, N., \& Constable, R. T. 2008. Anesthetic effects on regional CBF, BOLD, and the coupling between task-induced changes in CBF and BOLD: An fMRI study in normal human subjects. Magn. Reson. Med. 60, 987-996.

Rooney, N. J., \& Cowan, S. 2011. Training methods and owner-dog interactions: Links with dog behaviour and learning ability. Appl. Anim. Behav. Sci. 132, 169-177.

Rorden, C., \& Karnath, H. O. 2004. Using human brain lesions to infer function: a relic from a past era in the fMRI age? Nat. Rev. Neurosci. 5, 812-819.

Sachdev, R. N., Champney, G. C., Lee, H., Price, R. R., Pickens, D. R., Morgan, V. L., ... \& Ebner, F. F. 2003. Experimental model for functional magnetic resonance imaging of somatic sensory cortex in the unanesthetized rat. Neuroimage 19, 742-750.

Schultz, W., Apicella, P., Scarnati, E., \& Ljungberg, T. 1992. Neuronal activity in monkey ventral striatum related to the expectation of reward. J. Neurosci. 12, 4595-4610.

Smith, S. M., Jenkinson, M., Woolrich, M. W., Beckmann, C. F., Behrens, T. E., Johansen-Berg, H., ... \& Matthews, P. M. 2004. Advances in functional and structural MR image analysis and implementation as FSL. Neuroimage 23, S208-S219.

Stoewer S, Goense J, Keliris GA, Bartels A, Logothetis NK, et al. 2012 An analysis approach for high-field fMRI data from awake non-human primates. PLoS One 7 1-13.

Tsao, D. Y., Freiwald, W. A., Tootell, R. B., \& Livingstone, M. S. 2006. A cortical region consisting entirely of face-selective cells. Science $311,670-674$.

Udell, M. A., Dorey, N. R., \& Wynne, C. D. 2008. Wolves outperform dogs in following human social cues. Anim. Behav. 76, 1767-1773.

van den Berg, S. M., Heuven, H. C., van den Berg, L., Duffy, D. L., \& Serpell, J. A. 2010. Evaluation of the C-BARQ as a measure of stranger-directed aggression in three common dog breeds. Appl. Anim. Behav. Sci. 124, 136-141.

Van Den Heuvel, M. P., \& Pol, H. E. H. 2010. Exploring the brain network: a review on resting-state fMRI functional connectivity. Eur. Neuropsychopharmacol.20, 519-534. 
van der Meer, M. A., \& Redish, A. D. 2011. Theta phase precession in rat ventral striatum links place and reward information. J. Neurosci. 31, 2843-2854.

Van der Linden, A., Van Camp, N., Ramos-Cabrer, P., \& Hoehn, M. 2007. Current status of functional MRI on small animals: application to physiology, pathophysiology, and cognition. NMR. Biomed. 20, 522545.

Vermeire, S., Audenaert, K., Dobbeleir, A., De Meester, R., Vandermeulen, E., Waelbers, T., \& Peremans, K. 2009. Regional cerebral blood flow changes in dogs with anxiety disorders, measured with SPECT. Brain Imaging Behav. 3, 342-349

Vermeire, S., Audenaert, K., De Meester, R., Vandermeulen, E., Waelbers, T., De Spiegeleer, B., ... \& Peremans, K. 2011. Neuro-imaging the serotonin 2A receptor as a valid biomarker for canine behavioural disorders. Res. Vet. Sci. 91, 465-472.

Vermeire, S., Audenaert, K., De Meester, R., Vandermeulen, E., Waelbers, T., De Spiegeleer, B., ... \& Peremans, K. 2012. Serotonin 2A receptor, serotonin transporter and dopamine transporter alterations in dogs with compulsive behaviour as a promising model for human obsessive-compulsive disorder. Psychiatry Res. Neuroimaging 201, 78-87.

Waelbers, T., Polis, I., Vermeire, S., Dobbeleir, A., Eersels, J., De Spiegeleer, B., ... \& Peremans, K. 2015. Effect of ketamine on the regional cerebral blood flow and binding index of the 5-HT2A receptor radioligand 123 I-R91150 in the canine brain. Journal of Veterinary Behavior: Clinical Applications and Research. 10, 332-337.

Williams, M., \& Johnston, J. M. 2002. Training and maintaining the performance of dogs (Canis familiaris) on an increasing number of odor discriminations in a controlled setting. Appl. Anim. Behav. Sci. 78, 5565.

Willis, C. K., Quinn, R. P., McDonell, W. M., Gati, J., Parent, J., \& Nicolle, D. 2001. Functional MRI as a tool to assess vision in dogs: the optimal anesthetic. Vet. Opthalmol. 4, 243-253.

Yu, X., Tu, L., Lei, P., Song, J., Xu, H., \& Hou, X. 2014. Antiemesis effect and brain fMRI response of gastric electrical stimulation with different parameters in dogs. Neurogastroenterol. Motil. 26, 1049-1056. 
Figure Captions

Figure 1. Presentation of Visual Stimuli. A dog stationed in the receiver coil in the MRI bore views a hand signal from a human (left) and an on-screen visual stimulus (right).

Figure 2. Caudate Activation to Reward Versus No-Reward Signals. Anatomically defined regions of interest are shown in red in the coronal (A) and transverse (B) planes. (C) Unthresholded group mean activation to reward versus no-reward conditions in 12 dogs shown in the transverse (left) and coronal (middle) planes. Result thresholded at $\mathrm{P}=0.005$ also shown in the coronal plane (right). Color indicates tstatistic at each voxel against the null hypothesis of equal activity to reward and no-reward conditions.

Figure 3. Brain Activation to Familiar and Unfamiliar Social Olfactory Stimuli. Unthresholded group mean activation to scented stimuli (left) and the scent of familiar versus unfamiliar humans and dogs (right). Color indicates t-statistic at each voxel against the null hypothesis of equal activity to reward and no-reward conditions. Maximum activation to olfactory stimuli was in the olfactory cortex. Activation in the ventral caudate was higher for familiar than unfamiliar olfactory stimuli.

Figure 4. Auditory Activations to Human and Dog Vocalizations. (A) Representations of soundsensitive brain regions in dogs, presented on a rendered three dimensional brain. c, caudal; m, middle; $r$, rostral; ESG, ectosylvian gyrus; ESS, ectosylvian sulcus; SF, Sylvian fissure; SG, Sylvian gyrus; SSS, suprasylvian gyrus; and TP, temporal pole. (B) Auditory regions in the dog brain, defined as areas showing maximum activation to sound stimuli versus silence. FEW corrected the cluster level, using the uncorrected voxel threshold $p<0.001$ for dogs. Color represents t-statistics. (C) Auditory regions coded by maximum activation to human, dog, and non-vocal auditory stimuli. Figure adapted by permission from Andics et al., 2014.

Figure 5. Differential Activation to Visual Stimuli in the Dog Face Area and Primary Visual Cortex. (A) Individual regions for the dog face area (DFA) and primary visual cortex (V1) defined in each of 6 dogs during initial presentation of face and non-face stimuli. Each color represents the ROI of one dog. DFA was identified by the contrast of faces versus objects. V1 was identified by the average of all visual conditions relative to baseline (no stimulus presentation). Average signal change across all subjects in a second stimulus presentation phase (independent from initial presentation in which regions were defined) are shown to different visual stimulus categories in the DFA (B) and V1 (C). DFA showed a significant category effect $(F(3,24)=3.79, p=0.02)$, with a significantly greater response to images of faces compared to objects $\left({ }^{* *} p=0.004\right)$ and a marginally greater response to scenes $\left({ }^{*} p=0.06\right)$. (B) $\mathrm{V} 1$ had a similar level of response to all stimulus categories $(F(3,24)=0.42, p=0.74)$, and crucially was significantly different from DFA in face selectivity (i.e., faces compared to objects) $(F(1,30)=6.68, p=$ 0.02). 

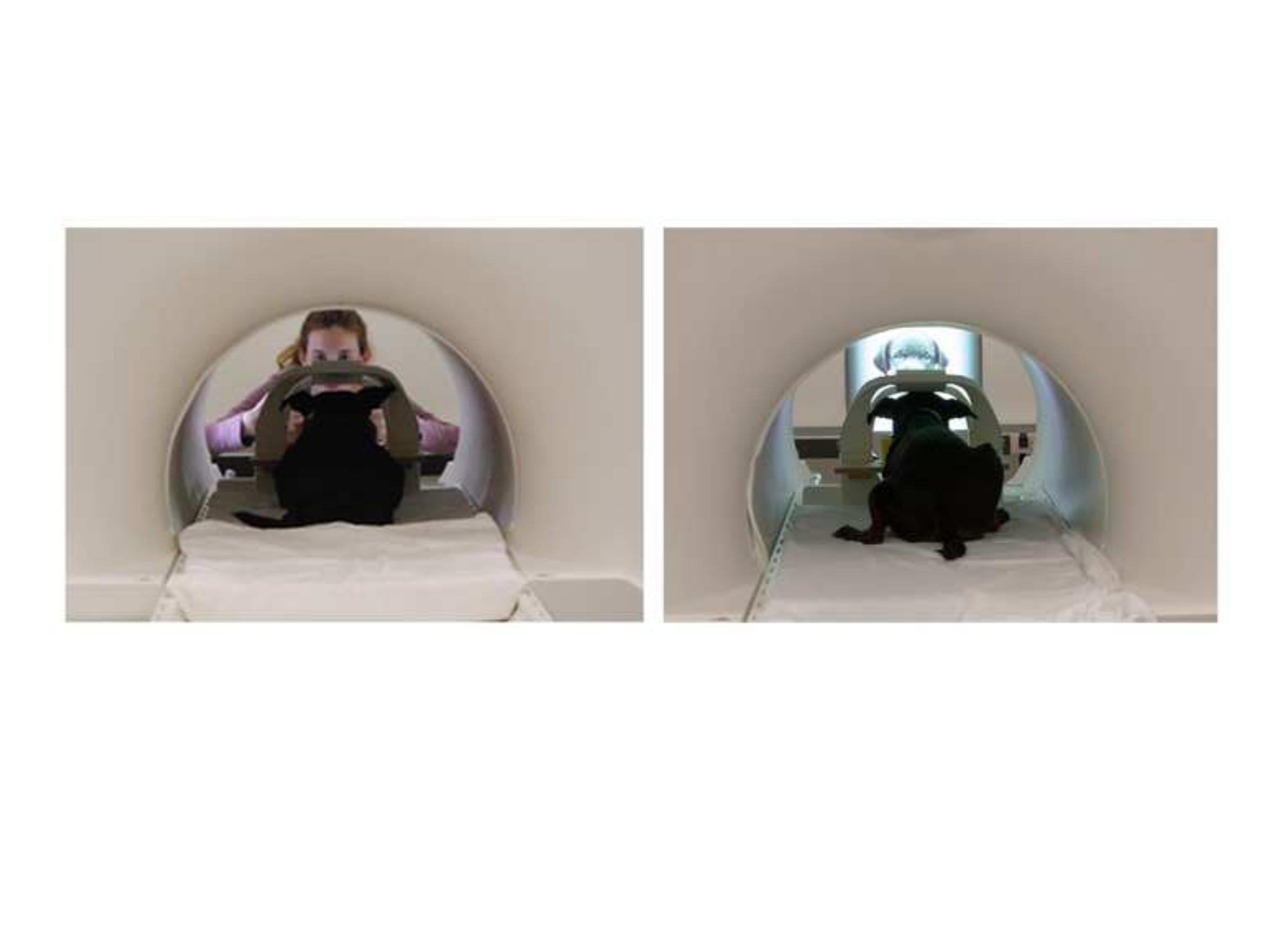

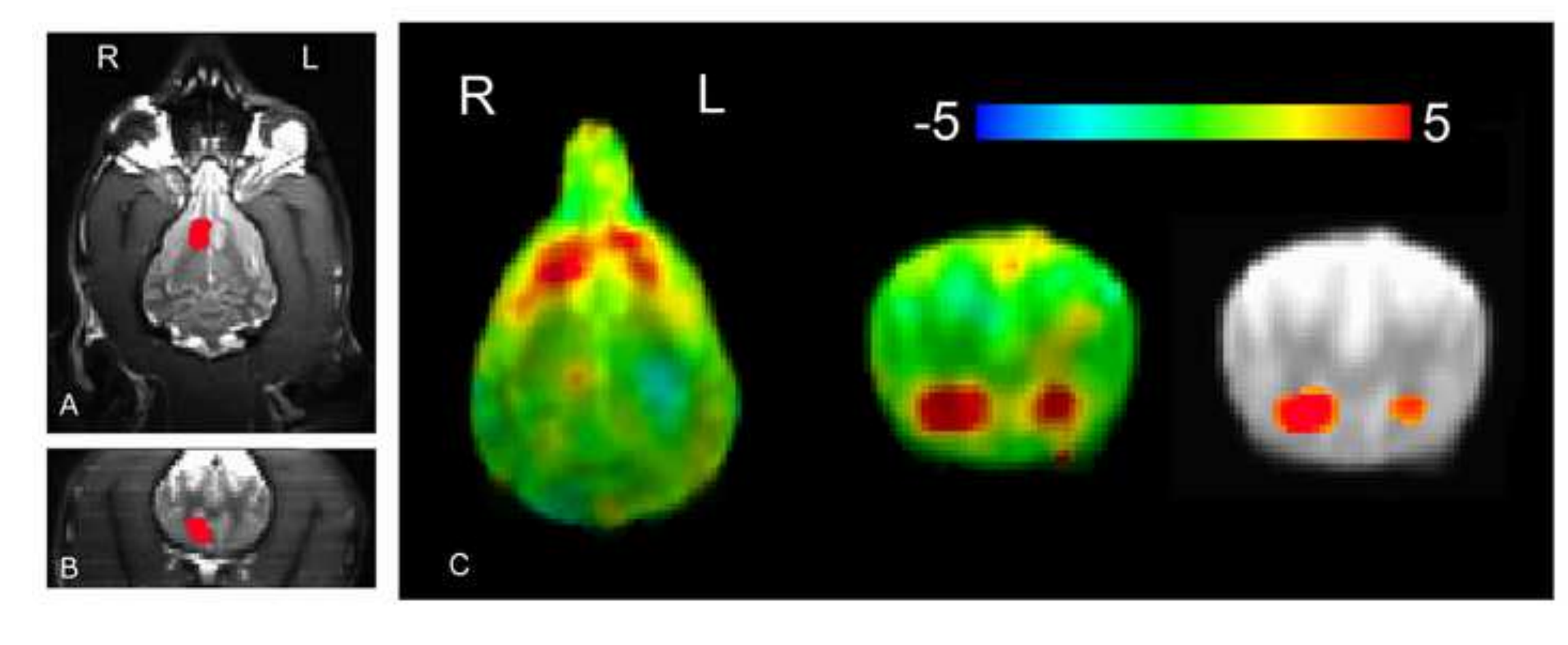

$-5$

5

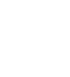

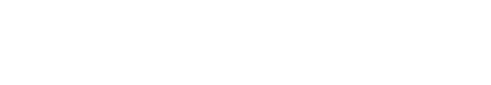

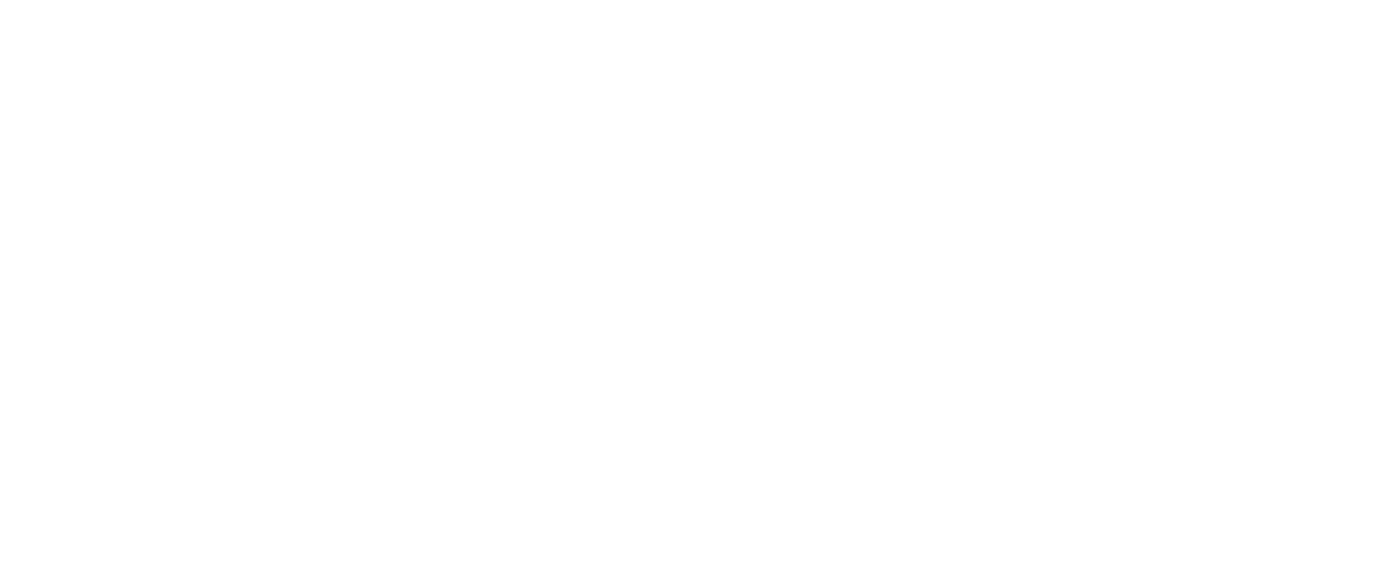
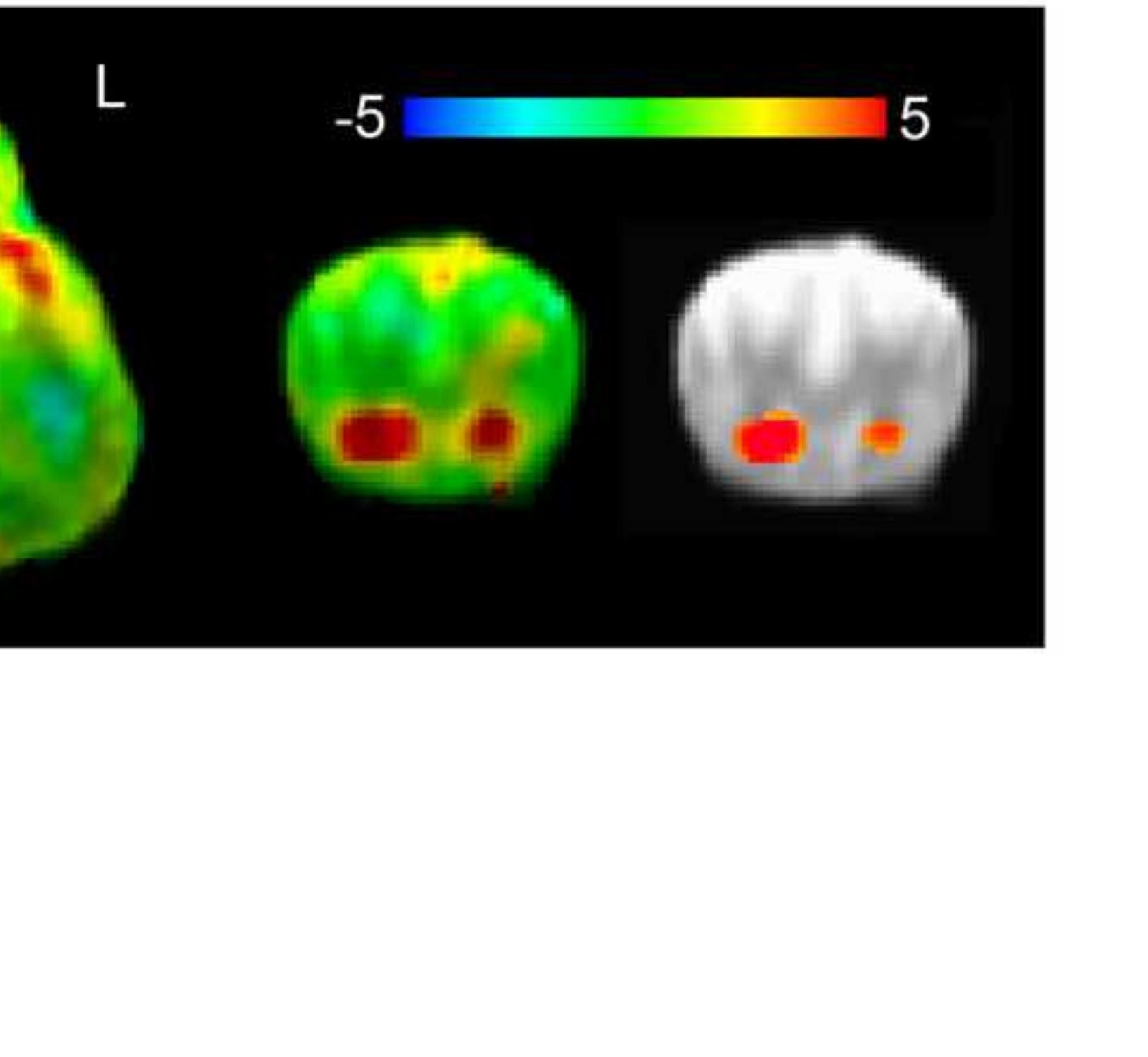
R

L

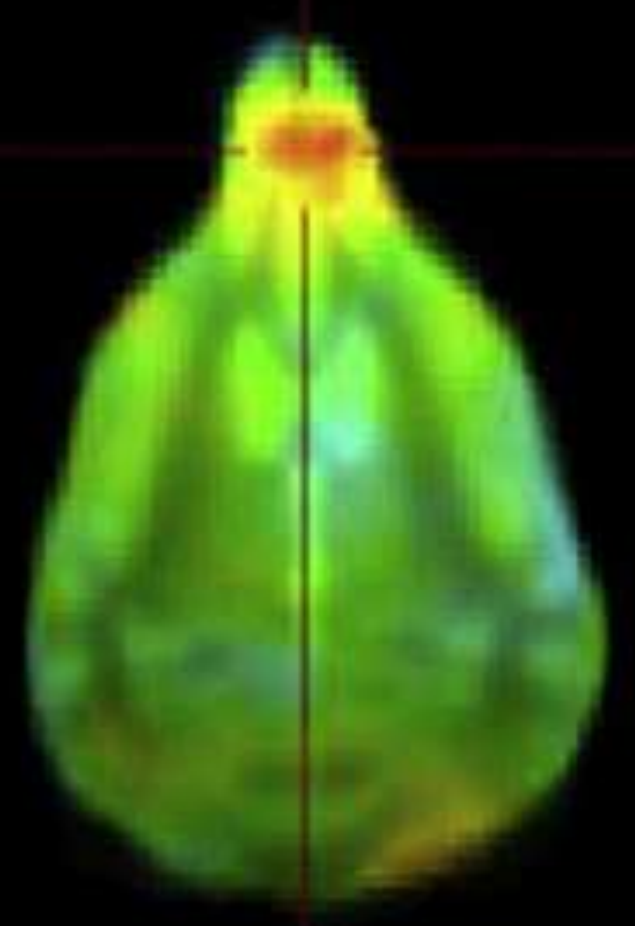

Neutral

$-4$
Scent
$\mathrm{R}$ L

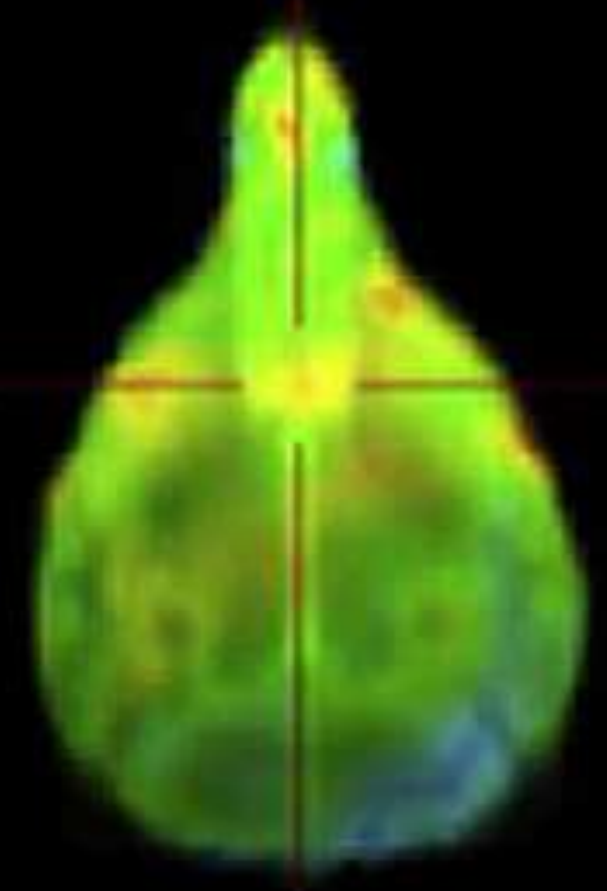

Strange

Familiar

\begin{tabular}{l|l} 
Neutral Scent \\
-4 &
\end{tabular}


A

\section{Dog brain}

B

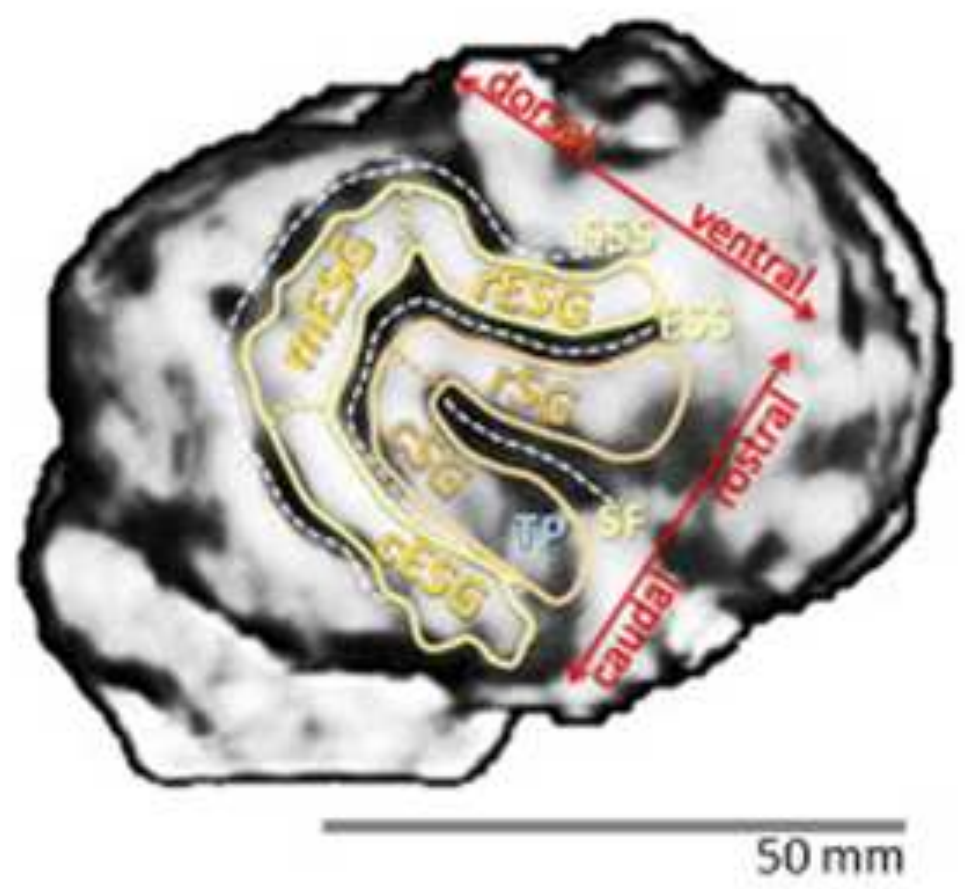

Auditory regions in dogs
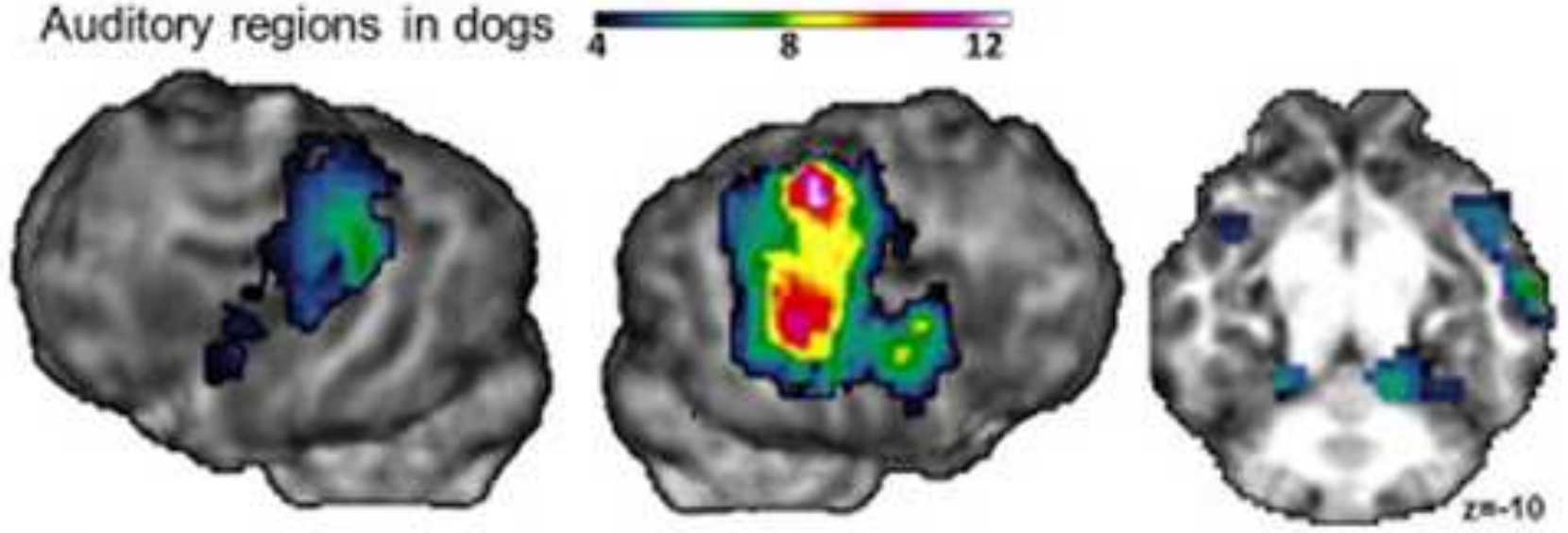

C

$$
\operatorname{MAX}=\text { human } \square \quad \operatorname{dog} \square \quad \text { non-vocal } \square
$$
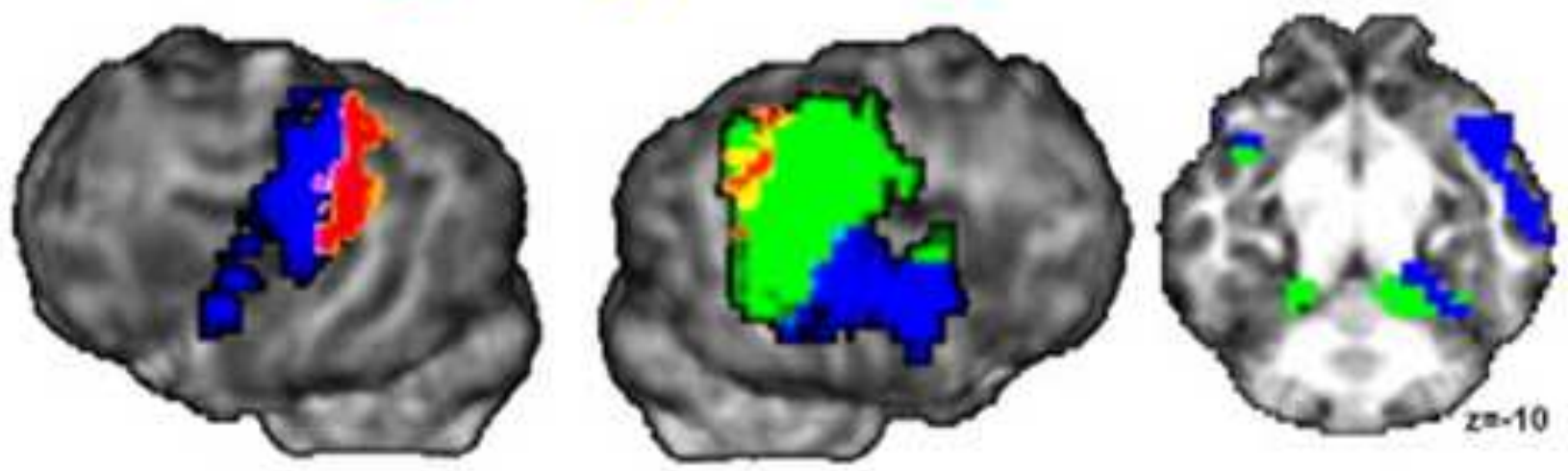
A

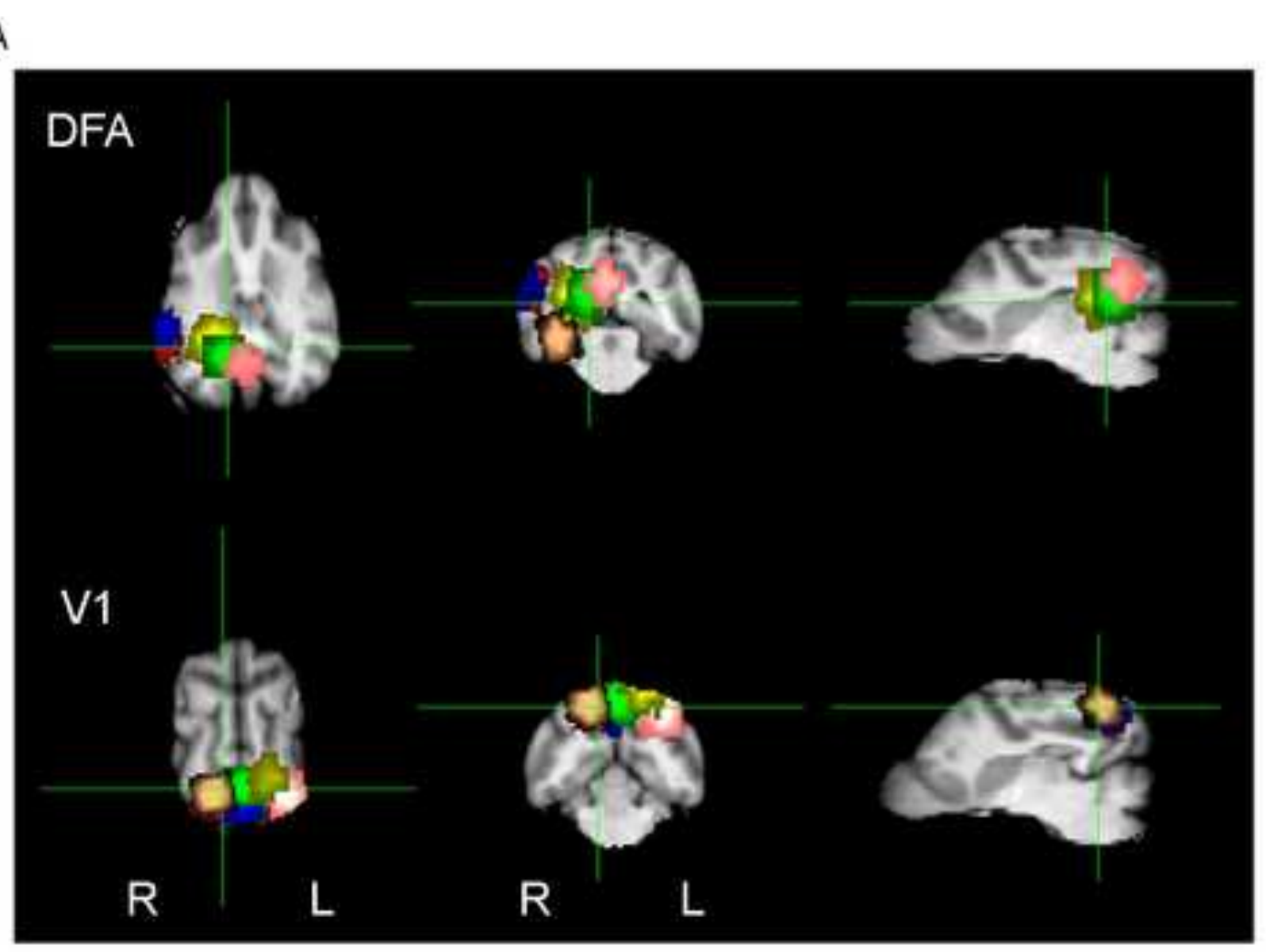

B

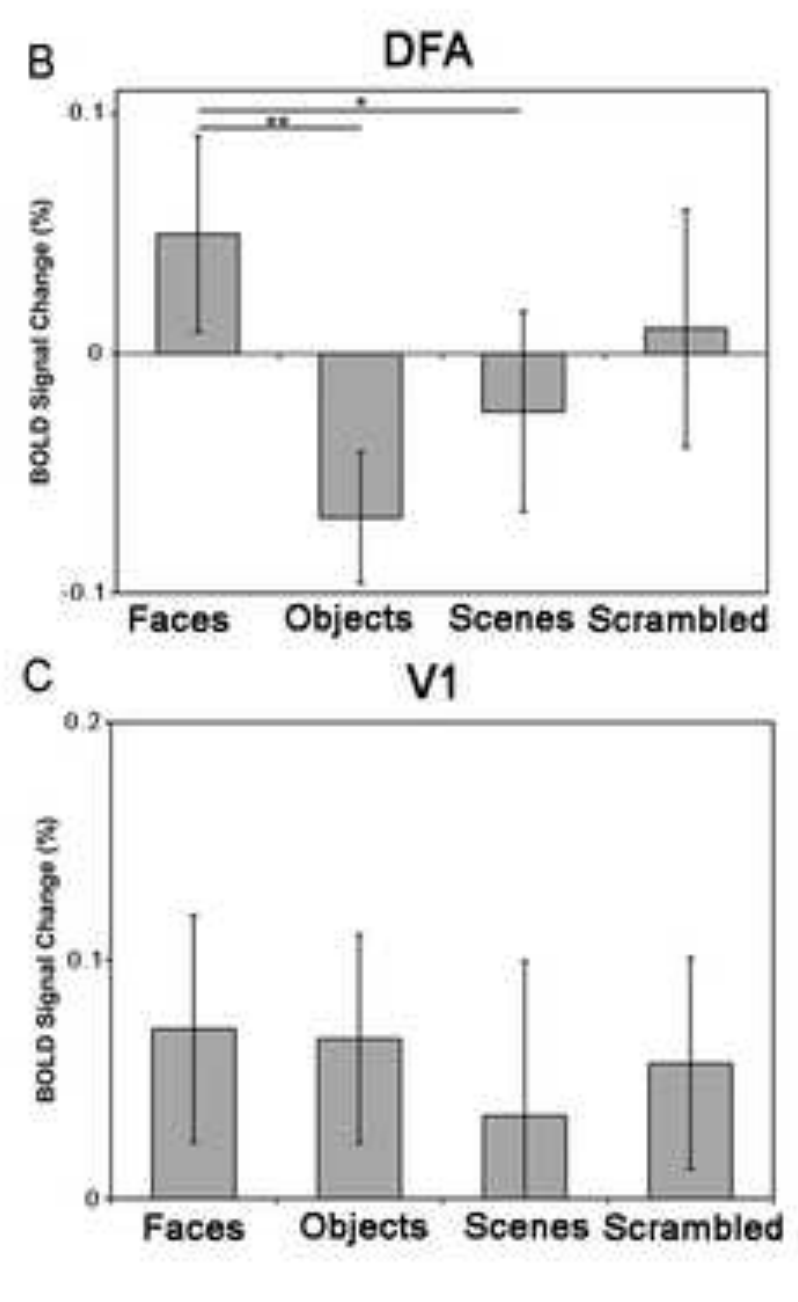

C

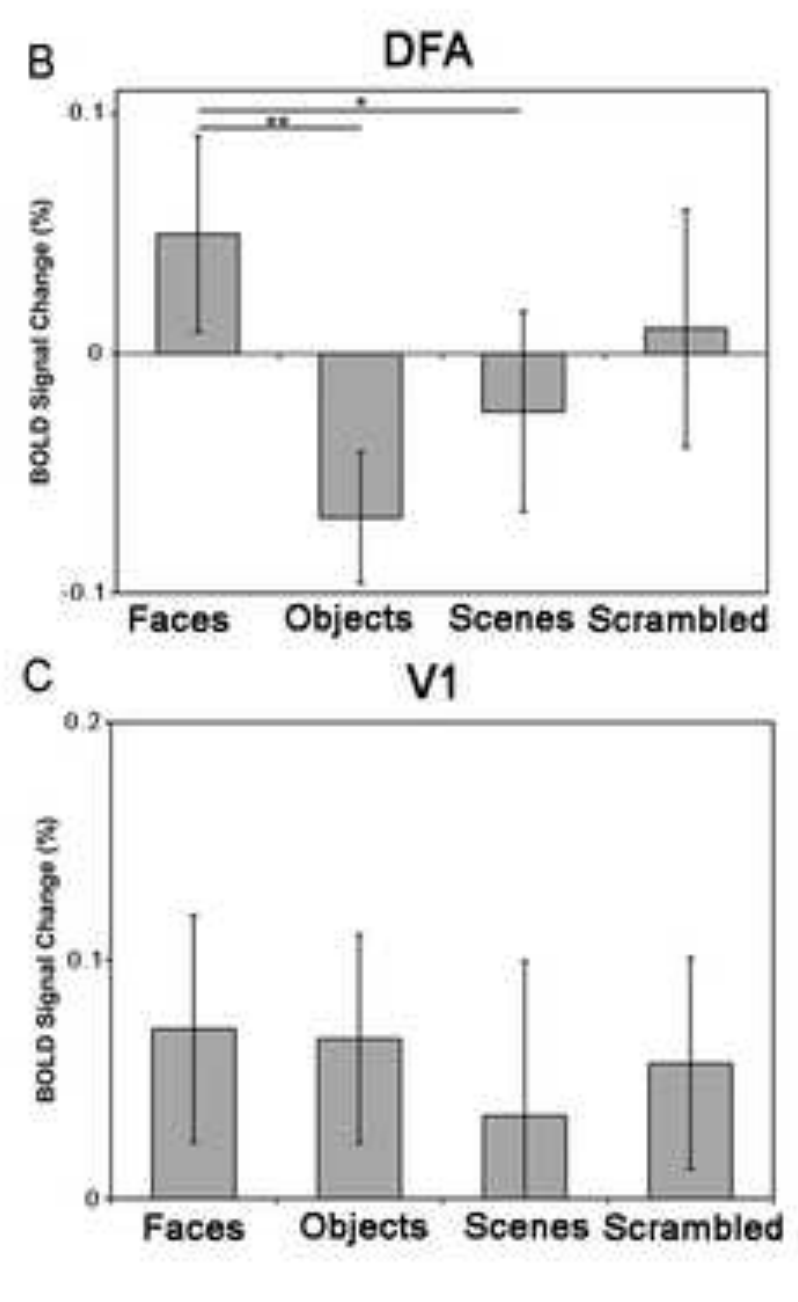$$
\text { - }
$$ 